Research Paper

\title{
Divergent Effect of Dezocine, Morphine and Sufentanil on Intestinal Motor Function in Rats
}

\author{
Xiaocui Bian $1^{*}$, Renlong Zhou ${ }^{1^{*}}$, Yuting Yang ${ }^{*}$, Peiying Li $^{1}$, Yannan Hang ${ }^{1}$, Youmin Hu ${ }^{2}$, Liqun Yang ${ }^{1 凶}$ \\ and Daxiang Wen $1{ }^{\bowtie}$ \\ 1. Department of Anesthesiology, Renji Hospital, School of Medicine, Shanghai Jiaotong University, Shanghai 200001, China; \\ 2. Laboratory Room for Physiology, Pathophysiology \& Pharmacology, School of Medicine, Shanghai Jiaotong University, Shanghai 200025, China \\ * Drs Bian X, Zhou R and Yang Y contributed equally to this work \\ $\triangle$ Corresponding authors: Prof. Wen DX wdxrwj@126.com \& Dr. Yang LQ lqyang72721@126.com, Department of Anesthesiology, Renji Hospital, \\ Shanghai Jiaotong University School of Medicine, Shanghai 200001, China.
}

( 2015 Ivyspring International Publisher. Reproduction is permitted for personal, noncommercial use, provided that the article is in whole, unmodified, and properly cited. See http://ivyspring.com/terms for terms and conditions.

Received: 2015.05.06; Accepted: 2015.08.17; Published: 2015.10.15

\begin{abstract}
Background: Opioid induced bowel dysfunction is the most common side effect of preoperatively administrated morphine, fentanyl and its derivative. However, the influence of dezocine on intestinal mobility is rarely reported. This study was designed to investigate the effects of dezocine, morphine and sufentanil on both intestinal smooth muscle contraction and propulsion in rats.

Methods: Contractile tension and frequency of isolated rat small intestine smooth muscle were measured using tension transducer after incubation with different concentrations of dezocine, morphine and sufentanil. The propulsive rate of methylene blue in rat intestinal tract was measured 30 minutes after intraperitoneal injection of morphine, sufentanil and dezocine. Percent of change in contractile tension and contraction frequency compared to baseline level were calculated to evaluate muscle contraction. Propulsive rate of methylene blue was calculated as the percentage of methylene blue moving distance in intestinal tract compared to the length of the small intestine.

Results: Morphine and sufentanil significantly increased the contractile tension of isolated small intestine smooth muscle at high doses. The contraction frequency did not change significantly among the 3 tested doses. Increasing the dose of dezocine from $1.7 \mathrm{mg} \cdot \mathrm{L}^{-1}$ to $10.2 \mathrm{mg} . \mathrm{L}^{-1}$ did not change either the contractile tension or the contraction frequency. The propulsive rate of methylene blue in intestinal tract was significantly decreased after the treatment with morphine, sufentanil and dezocine $(45.6 \%, 43.7 \%$, and $42.1 \%$ respectively) compared to control group $(57.1 \%)$, while the difference among the 3 drug groups were not significant.

Conclusion: Morphine and sufentanil may dose dependently increase the contractile tension and contraction ability of isolated rat small intestine smooth muscle, while dezocine has no significant effect on intestine smooth muscle contraction. However, all these opioids might impair small intestinal propulsion.
\end{abstract}

Key words: dezocine; contractile tension; propulsive motility; small smooth muscle intestine

\section{Introduction}

Opioids treatment is the most widely prescribed medication for pain relief [1]. However, alone with its broad-spectrum analgesic properties, side effects such as nausea, cognitive impairment, addiction and bowel dysfunction may also occur [2]. In the intestine, opioids exert their function by acting on the enteric nervous system. They can bind to the myenteric and submucosal plexuses, causing dysmobility, decreased fluid secretion and sphincter dysfunction, all of which may lead to opioid induced bowel dysfunction (OIBD) [3]. OIBD is a clinically distressing condition which may persist indefinitely and manifest with 
different symptoms such as dry mouth, gastro-oesophageal reflux, vomiting, bloating, abdominal pain, anorexia, hard stool, constipation and incomplete evacuation [4]. These gastrointestinal symptoms add burden to the patients suffering from chronic pain, which also negatively impact the quality of life and, in some cases, alter the effect of opioid treatment itself [3, 5]. Generally, morphine, fentanyl and its derivatives are commonly used opioid agonists for perioperative pain management, nevertheless, studies have shown that constipation is the most common and painful adverse reaction of opioid agonists treating chronic pain. The incidence of constipation was reported as high as $40 \%$ [6], which largely limit the clinical use of opioids [7, 8]. Dezocine is an opioid receptor agonist - antagonist gaining popularity in clinical practice because of its mild side effects and high efficacy as an analgesic $[9,10]$. However, the influences of dezocine on intestinal mobility are rarely reported. Therefore, this study was designed to investigate the effects of dezocine, morphine and sufentanil on the mobility of intestinal smooth muscle in rats. Our findings may provide new evidence for reasonable selection of opioids to avoid OBID in post-operative pain management [11].

\section{Materials and methods}

\section{Animals}

This study was approved by the Institutional Animal Care and Use Committee, Jiao-tong University School of Medicine. Male Sprague-Dawley rats, weighing 180-200 g, purchased from the Chinese Academy of Sciences Shanghai Laboratory Animals Center (SLAC), were housed in a temperature and humidity-controlled environment with a 12-h light dark cycle. The rats received unlimited access to water and food. The food was removed from the cage 16 hours prior to the experiments.

\section{Procedures} below.

The study consisted two series of experiments as

Series 1. The effects of different opioids on the contractile tension of isolated small intestine smooth muscle from rats

Preparation and installation of isolated intestine was prepared according to methods described previously [12]. Briefly, animals were sacrificed by cervical dislocation, then laparotomy was quickly carried out and the mesenteric edge was separated along to remove the ileum and put into the Kreb's solution. Mesenteric and peripheral adipose tissues were removed carefully and the intestinal contents were cleared. The intestinal segments were cut into 1 to 1.5 $\mathrm{cm}$ pieces, and the rest of the intestinal segments were set in the spare Kreb's liquid. All of the bowel segments were put separately in $20 \mathrm{ml} K \mathrm{Kreb}^{\prime}$ s liquid baths. Both ends were threaded diagonally. The upper end was connected to the tension transducer (Shanghai Jide teaching experiment equipment factory, Shanghai, China), while the lower end was fixed to the L-shaped hook. The temperature was set at $(37 \pm$ $0.5){ }^{\circ} \mathrm{C}$ thermostat and a mixed gas of $95 \% \mathrm{O}_{2}+5 \%$ $\mathrm{CO}_{2}$ at 60 to 80 bubbles per minute was passed into the bath. The intestinal bowel tension transducer was connected to the powerlab/8sp biological function experimental system (Australia AD Instrument Pty Ltd company, Australia) while the contractile activities of the intestinal were recorded. The isolated intestinal muscle was given a $1.0 \mathrm{~g}$ load, and stabled for 30 min. Morphine hydrochloride (Shenyang First Pharmaceutical Factory, Shanghai, China) was added to a constant temperature water bath consisting of 20 $\mathrm{ml}$ of Kreb's solution, and the final concentration was $5 \mathrm{mg}$.L-1. Intestinal muscle tone and contraction frequency were recorded, then $10 \mathrm{mg} . \mathrm{L}^{-1}$ and $30 \mathrm{mg} . \mathrm{L}^{-1}$ of morphine hydrochloride were added after the medium was changed and stabled for 30 minutes. The above steps were repeated with escalating doses of 20,

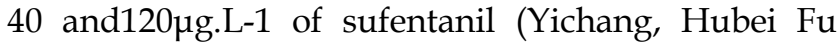
Pharmaceutical Co., Ltd, Hubei, China.) co-incubated, and 1.7, 3.4 and $10.2 \mathrm{mg} . \mathrm{L}-1$ of dezocine (Yangtze River Pharmaceutical Group Co.Ltd, Jiangsu, China.) respectively. Muscle tension and contraction frequency were measured and recorded before and after opioids treatment.

\section{Series 2. Intestinal propulsion assay in rats}

Animals were fasted for $24 \mathrm{~h}$, and were randomly distributed into 3 experimental groups and 1 control group. $1 \mathrm{ml}$ of saline was injected intraperitoneally in the control group while $1.04 \mathrm{mg} \cdot \mathrm{kg}^{-1}$ of morphine, $2.08 \mu \mathrm{g} . \mathrm{kg}^{-1}$ of sufentanil and $1.04 \mathrm{mg} \cdot \mathrm{kg}^{-1}$ of dezocine for treatment groups respectively with a total volume of $1 \mathrm{ml}$ in each. $2 \mathrm{~min}$ later, $1 \mathrm{ml}$ methylene blue (the Shanghai Exhibition cloud Chemical Co., Ltd.) solution was given orally. 30min later, the rats were sacrificed by cervical dislocation, the mesenterys were separated and the bowels pulled into a straight line. Take pylori as a starting point to measure the following data as moving distance of methylene blue in the intestinal tract (D) and small intestine length, pyloric to the ileocecal distance (L). The ratio of propulsive distance of intestine were calculated according to the formula: propulsive distance rate $(\mathrm{PDR})=\mathrm{D} / \mathrm{L} \times 100 \%$.

\section{Statistical Methods}

Data analysis was performed with a personal 
computer statistical software package (Prism version 4.0; Graph-Pad Software, San Diego, CA). Changes of the contractile tension and frequency to opioids were expressed as percentage of the baseline values and the data was presented as mean \pm SEM. Statistical analysis was performed with two-way repeated measure analysis of variance followed by the Bonferroni test. Data were considered statistically significant for $P$ values less than 0.05 .

\section{Result}

\section{Morphine and sufentanil, but not dezocine, dose-dependently increased the smooth mus- cle contraction of isolated small intestine from rats.}

In the morphine group, no difference was observed in the $5 \mathrm{mg} . \mathrm{L}^{-1}$ group $(P>0.05)$, but there were significantly increased muscle tension at concentrations of the 10 and $30 \mathrm{mg}$.L- $1(\mathrm{P}<0.05)$. In the sufentanil group, the muscle tone at concentration of 40 and $120 \mu \mathrm{g}$.L-1 but not $20 \mu \mathrm{g}$.L-1 were significantly higher than basal $(\mathrm{P}<0.05)$. While the differences of muscle tensions among all dose groups of dezocine were not statistically significant compared to basal value (P> 0.05). All three opioids morphine, sufentanil and dezocine, had no significant difference on the contractile frequency of the isolated rat small intestine smooth muscle compared to basal (shown in Figure 1).

\section{Morphine, sufentanil and dezocine unexcep- tionally impaired small intestinal propulsive motility in rats}

Our results showed significant decrease in propulsive distance rate marked by methylene blue in morphine, sufentanil and dezocine groups compared to those in the control group $(P<0.05)$. Furthermore there were no difference among these 3 treatment groups, $(P>0.05$, shown in Figure 2$)$.

\section{Discussion}

The results of this study confirmed that morphine and sufentanil dose dependently increased the intestinal smooth muscle contractility in rats, while dezocine did not cause significant change in intestine smooth muscle contraction. Although all of these opioids impaired the propulsive motility of small intestine. This study provided experimental evidence that dezocine, a mixed opioid receptor agonist-antagonist, might have fewer intestinal side effects compared to conventional opioids, such as morphine and sufentanil.

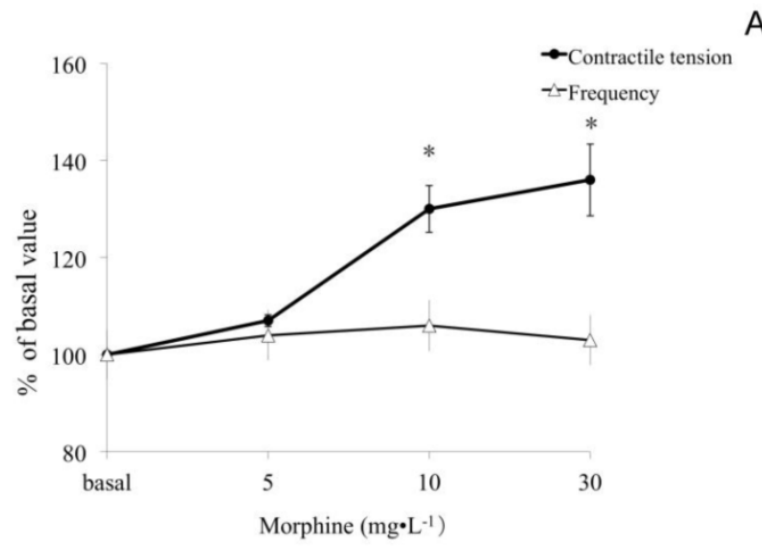

A

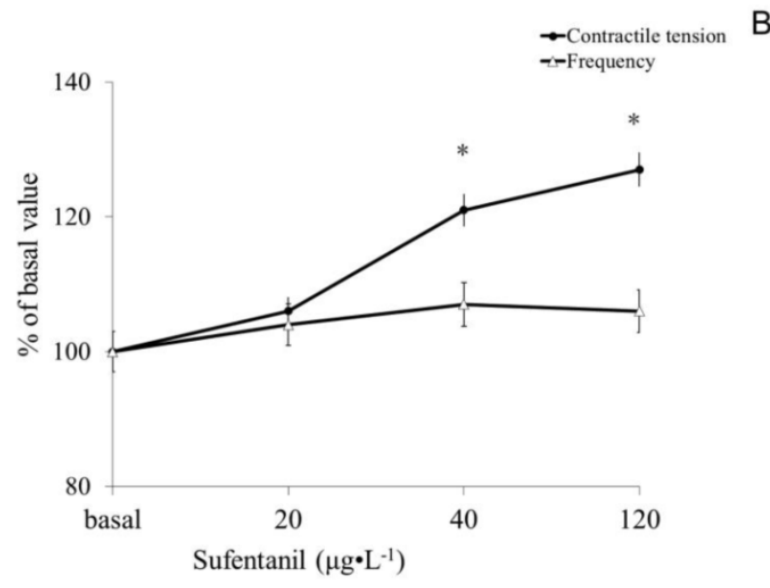

B

C

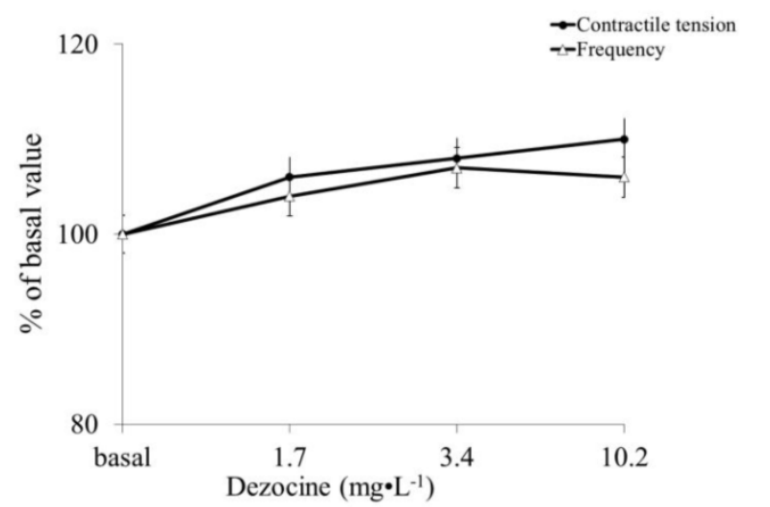

Figure 1. Effect of morphine, sufentanil and dezocine on contractile tension and frequency of the isolated small intestine smooth muscle of rats. There was no difference in muscle contractile tension after $5 \mathrm{mg}$.L-1 of morphine incubation compared to those of baseline, $(P>0.05)$, while there were significantly increased muscle tension at concentrations of the 10 and $30 \mathrm{mg}$.L-1 group $(P<0.05$, figure $1 \mathrm{~A})$. The muscle tone in 40 and $120 \mu \mathrm{g}$.L-1 but not $20 \mu \mathrm{g}$.L- 1 of sufentanil group were significantly higher than those of baseline $(P<0.05$, figure $1 B)$. The differences in muscle tensions among all dose groups of dezocine were not statistically significant compared to basal value ( $P>0.05$, figure $1 C)$. Opioids whether morphine, sufentanil or dezocine, had no significant differences in the contractile frequency of the isolated small intestine smooth muscle of rats compared to basal values $(P>0.05)$. Values are means \pm SEM; $n=10$ in each group; percent change in a given variable compared with its baseline; the baseline values and any opioids groups were compared by two-way analysis of variance (ANOVA) using the Bonferroni test. ${ }^{*} p<0.05$, vs the basal values. 


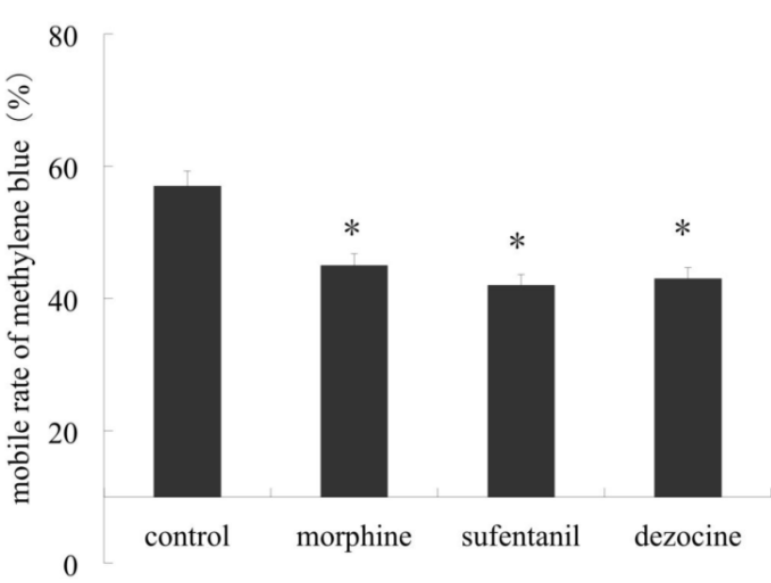

Figure 2. Effect of morphine, sufentanil and dezocine on propulsive motility of small intestine of rats. Animals were injected intraperitoneally with $1.04 \mathrm{mg} . \mathrm{kg}-1$ of morphine, $2.08 \mu \mathrm{g} . \mathrm{kg}-1$ of sufentanil and $1.04 \mathrm{mg} . \mathrm{kg}-1$ of dezocine for treatment groups respectively and $1 \mathrm{ml}$ saline as control. $30 \mathrm{~min}$ after $1 \mathrm{ml}$ methylene blue was given orally the rats were sacrificed and the bowels pulled into a straight line. Our result showed a significant decrease in propulsive distance rate in morphine, sufentanil and dezocine groups compared to those in control group $(P<0.05)$, but there were no difference in advance rate among these 3 treatment groups, $(P>0.05)$. * $P$ $<0.05$, vs control.

Pain usually has negative impacts on the living quality of patients and opioids represent the most widely prescribed analgesics all over the world because of its high efficacy as analgesics[13]. However, the accompanying adverse effects of opioids are not negligible and inevitably limit its clinical use[2]. Opioid induced bowel dysfunction, $\operatorname{OIBD}[14,15]$ is the most common side effect and manifests as nausea, vomiting, gastro-oesophageal reflux, constipation. Morphine and sufentanil, although widely used in clinical settings for pain management, are often reported to cause OIBD. Dezocine is an opioid receptor agonist - antagonist gaining popularity among practitioners because of its mild side effects and high efficacy $[6,9,16]$. So far, little literature has focused on their adverse effects on bowel function, which constitutes the major factor that limit the clinical use of opioids. Therefore, our study was designed to investigate the influence of these three opioids on intestinal mobility in rats.

Opioids inhibit intestinal mobility by acting on opioid receptors[17], which are located both in the central nervous system and in peripheral organs, such as the digestive tract. Gastrointestinal opioid receptors are located in the wall of the stomach, small intestine, large intestine, neurons and smooth muscle[18, 19]. Thus, systematical administration of opioids exert their effects unexceptionally on these peripheral receptors[20]. Researchers have proposed several possible mechanisms underlying this multi-channel and complex process. Firstly, opioid agonists may inhibit the neuronal excitability of enteric nervous system and reduce its neurotransmitter re- lease,[21] including acetylcholine and substance $\mathrm{P}[22]$. Secondly, morphine may increase the still contraction tension of intestinal smooth muscle, and inhibit gastrointestinal peristalsis wave and intestinal migrating motor complex (MMC), which was partialy consistent with our present results[20]. Thirdly, opioids reduced intestinal secretion, leading to formation of dry, hard stool in the large intestine[5]. Finally, opioids also acts on opioid receptors in the central nervous system, which could inhibit intestinal function through activation of the presynaptic alpha receptor to inhibit enteric nervous system neurotransmitter release [11].

As a mixed agonist - antagonist, dezocine mainly activate the kappa receptor, which generate sedative and analgesic effects. However, the kappa receptor agonist does not inhibit gastric emptying and small intestinal motility after intraperitoneal injection[23], while it inhibits the contraction of the colon by acting on the central[18, 24]. It was observed that dezocine did not elevate the muscle tone of the ileocecal sphincter in cats[10]. Consistently, our study demonstrated that dezocine caused a slight increase in intestinal smooth muscle contraction and frequency in vitro. But we also found that dezocine inhibited the intestinal propulsive motility when injected intraperitoneally in vivo. This phenomena can be explained by the previous finding that the kappa receptor agonist can act on intestinal smooth muscle strips in vitro, but does not affect the spontaneous contraction of the muscle strips[17],[24]. In addition to the kappa receptor agonist effect, dezocine is also a mixed opioid $\mathrm{mu}$ receptor agonist and antagonist. It partially agonizes the $\mu$ opioid receptor and simultaneously antagonizes the $\mu$ receptor[10, 25]. This may also help to explain its negative effect on intestinal propulsive motility.

In conclusion, our study find that classic opioid receptor agonists, such as morphine and sufentanil, dose dependently enhance the contraction of intestine smooth muscle in rats. But dezocine, an opioid receptor mixed agonist - antagonist, does not cause obvious effects on intestine smooth muscle contraction. Moreover, we also showed that dezocine could partially impair the bowel motor function by inhibiting the propulsive motility of small intestinal in rats. Therefore, this experimental study indicate that, compared to morphine and sufentanil, dezocine may cause lighter intestinal adverse effects in the use of pain-management.

\section{Acknowledgement}

This study was supported by National Natural Science Foundation Committee (NSFC) of China (Grant No. 81272066 and No. 81471846). 


\section{Competing Interests}

The authors have declared that no competing interest exists.

\section{References}

1. Trescot AM, Glaser SE, Hansen H, Benyamin R, Patel S, Manchikanti L. Effectiveness of opioids in the treatment of chronic non-cancer pain. Pain Physician. 2008; 11: S181-200.

2. Ahlbeck K. Opioids: a two-faced Janus. Curr Med Res Opin. 2011; 27: 439-48.

3. Kurz A, Sessler DI. Opioid-induced bowel dysfunction: pathophysiology and potential new therapies. Drugs. 2003; 63: 649-71.

4. Pappagallo M. Incidence, prevalence, and management of opioid bowel dysfunction. Am J Surg. 2001; 182: 11S-8S.

5. Holzer P. Treatment of opioid-induced gut dysfunction. Expert Opin Investig Drugs. 2007; 16: 181-94

6. Minkowitz HS, Singla NK, Evashenk MA, Hwang SS, Chiang YK, Hamel LG, et al. Pharmacokinetics of sublingual sufentanil tablets and efficacy and safety in the management of postoperative pain. Reg Anesth Pain Med. 2013; 38: 131-9.

7. Brock C, Olesen SS, Olesen AE, Frokjaer JB, Andresen T, Drewes AM. Opioid-induced bowel dysfunction: pathophysiology and management. Drugs. 2012; 72: 1847-65.

8. Liu YF, Chen KB, Lin HL, Ho CH, Liu SK, Liu YC, et al. Comparison of the effect of epidural and intravenous patient-controlled analgesia on bowel activity after cesarean section: a retrospective study of 726 Chinese patients. Acta Anaesthesiol Taiwan. 2009; 47: 22-7.

9. Pandit UA, Kothary SP, Pandit SK. Intravenous dezocine for postoperative pain: a double-blind, placebo-controlled comparison with morphine. J Clin Pharmacol. 1986; 26: 275-80.

10. Ouyang A. The partial opiate receptor agonists, dezocine and ciramadol act as mu receptor antagonists at the feline ileocecal sphincter. Life Sci. 1989; 45: 1491-7.

11. Bueno L, Fioramonti J. Action of opiates on gastrointestinal function. Baillieres Clin Gastroenterol. 1988; 2: 123-39.

12. Feng ZY, Mao LG, Lu Y. [Effect of morphine chloride on contractility of small intestinal muscle in vitro or in vivo and its mechanisms]. Zhejiang Da Xue Xue Bao Yi Xue Ban. 2008; 37: 271-5.

13. Junker U, Lux EA, Neugebauer EA, Basler HD. [Therapy of pain in multiborbid elderly patients]. Med Monatsschr Pharm. 2009; 32: 175-81.

14. McNicol ED, Boyce D, Schumann R, Carr DB. Mu-opioid antagonists for opioid-induced bowel dysfunction. Cochrane Database Syst Rev. 2008: CD006332.

15. Ross GR, Gabra BH, Dewey WL, Akbarali HI. Morphine tolerance in the mouse ileum and colon. J Pharmacol Exp Ther. 2008; 327: 561-72.

16. Cohen RI, Edwards WT, Kezer EA, Ferrari DA, Liland AE, Smith ER. Serial intravenous doses of dezocine, morphine, and nalbuphine in the management of postoperative pain for outpatients. Anesth Analg. 1993; 77: 533-9.

17. Poonyachoti S, Portoghese PS, Brown DR. Characterization of opioid receptors modulating neurogenic contractions of circular muscle from porcine ileum and evidence that delta- and kappa-opioid receptors are coexpressed in myenteric neurons. J Pharmacol Exp Ther. 2001; 297: 69-77.

18. Chamouard P, Klein A, Martin E, Adloff M, Angel F. Regulatory role of enteric kappa opioid receptors in human colonic motility. Life Sci. 1993; 53: 1149-56.

19. Brown DR, Poonyachoti S, Osinski MA, Kowalski TR, Pampusch MS, Elde RP, et al. Delta-opioid receptor mRNA expression and immunohistochemical localization in porcine ileum. Dig Dis Sci. 1998; 43: 1402-10.

20. Manara L, Bianchetti A. The central and peripheral influences of opioids on gastrointestinal propulsion. Annu Rev Pharmacol Toxicol. 1985; 25: 249-73.

21. Holzer P. Opioids and opioid receptors in the enteric nervous system: from a problem in opioid analgesia to a possible new prokinetic therapy in humans. Neurosci Lett. 2004; 361: 192-5.

22. Tavani A, Petrillo P, La Regina A, Sbacchi M. Role of peripheral mu, delta and kappa opioid receptors in opioid-induced inhibition of gastrointestinal transit in rats. J Pharmacol Exp Ther. 1990; 254: 91-7.

23. Liu R, Huang XP, Yeliseev A, Xi J, Roth BL. Novel molecular targets of dezocine and their clinical implications. Anesthesiology. 2014; 120: 714-23.

24. Menozzi A, Pozzoli C, Zullian C, Poli E, Serventi P, Bertini S. Inhibition of motility in isolated horse small intestine is mediated by kappa but not micro opioid receptors. Equine Vet J. 2012; 44: 368-70.

25. Picker MJ. Discriminative stimulus effects of the mixed-opioid agonist/antagonist dezocine: cross-substitution by mu and delta opioid agonists. J Pharmacol Exp Ther. 1997; 283: 1009-17. 